\title{
The structure function as a metric for roughness and figure
}

\author{
Robert E. Parks ${ }^{1 *}$ and Michael T. Tuell ${ }^{2}$ \\ ${ }^{1}$ Optical Perspectives Group, LLC, 7011 E. Calle Tolosa, Tucson, AZ 85750 \\ ${ }^{2}$ Steward Observatory, The University of Arizona, Tucson, Arizona 85721
}

\begin{abstract}
As optical designs become more sophisticated and incorporate aspheric and free form surfaces, the need to specify limits on mid-spatial frequency manufacturing errors becomes more critical, particularly as we better understand the effects of these errors on image quality. While there already exist methods based on Fourier analysis to specify these errors in most commercial interferometry software, the method of calculation and the power spectral density (PSD) results remain obscure to many in the optical design and manufacturing field.

We suggest that the structure functions (SF) contains the same information as in the Fourier based PSD but in a way that is much more transparent to analysis, interpretation and application as a specification. The units of measure are more familiar and the concept behind the analysis is simpler to understand. Further, the information contained in the structure function (or PSD) allows a complete specification of an optical surface from the finest measurable detail of roughness to the overall figure.

We discuss the origin of the structure function in the field of astronomy to describe the effects of air turbulence on image quality, the simple mathematical definition of the structure function and its easy means of calculation and how its results should be scaled depending on the location of the optical surface in a system from pupil to image plane. Finally, we give an example of how to write a specification of an optical surface using the structure function.
\end{abstract}

Keywords: Structure function, figure and roughness surface specification, power spectral density, PSD, fabrication, midspatial frequency errors

\section{INTRODUCTION}

A traditional specification for optical elements, based on the Rayleigh criterion, sets a limit of $\lambda / 4$ peak-to-valley (PV), but, as we will discuss, this is a relatively poor metric for precision optics. One reason is that visual inspection of Ronchigrams or interferograms, for example, includes human expertise, which can automatically reject noisy data. Computers are very literal, and must be programmed around noise inherent in a measurement. A simplistic PV computation from a wavefront map would include noise - the reported value is the difference between the maximum pixel value and the minimum, which can be vastly different from the PV that a human would report from a visual inspection of the same data. There are of course ways to program computers to alleviate this flaw, including a simple root-mean-square (rms) metric, $\mathrm{PVr}^{1}$, and by first rejecting the top and bottom $1 \%$ (for example) of extreme pixel values, but care must be taken with any PV reporting. The second reason is that while a wavefront may meet, even literally, a certain PV spec, the optic may not perform as desired ${ }^{2,3}$ primarily due to mid-spatial frequency errors. In recent years, this approach of viewing the wavefront or surface error as a function of spatial frequency has become more and more accepted, in one form or another, as a way to specify precision optics.

*Corresponding author: reparks@optiper.com

Optical System Alignment, Tolerancing, and Verification X, edited by José Sasián,

Richard N. Youngworth, Proc. of SPIE Vol. 9951, 99510J · (c) 2016 SPIE

CCC code: $0277-786 X / 16 / \$ 18 \cdot$ doi: $10.1117 / 12.2238600$

Proc. of SPIE Vol. $995199510 \mathrm{~J}-1$ 
Several methods have been proposed to segregate errors into spatial frequencies, including the well-known modulation transfer function (MTF) and optical transfer function (OTF), as well as auto-correlation, the power spectral density (PSD) and structure function (SF). This paper discusses the final two, the PSD and the SF, and their relationship.

\section{THE STRUCTURE FUNCTION}

The structure function is perhaps the least well known of these concepts, at least in the optical fabrication community, but the basic concept is easy to understand in mathematical terms, so it pays to give a little background. In astronomy the main factor affecting good image quality is the turbulent atmosphere and thus the desire to locate telescopes where the atmosphere is least turbulent. In 1941, the Russian mathematician, Andrei Kolmogorov, made a significant contribution to the study of turbulence 5 . He deduced that the turbulent atmosphere obeys a 5/3 power law, in contrast to the spectrum of Brownian motion, which obeys a 6/3 ( ${ }^{\text {nd }}$ power) law. One of Kolmogorov's students, Aleksandr Obukhov, coined the term structure function (SF).

$$
S F(r)=\left\langle\left(z\left(r+r^{\prime}\right)-z\left(r^{\prime}\right)\right)^{2}\right\rangle,
$$

that is the second moment of the difference ${ }^{5}$ between points separated by $r$. The angled brackets indicate the ensemble average over many points, $r$ '.

The next major step was made in the mid-1960's by David Fried, describing how the statistics of the turbulent atmosphere can be transferred to the statistics of a wavefront transmitted through the atmosphere. These effects on the wavefront depend on a spatial parameter, called the Fried parameter, which is 15-20 cm for "good seeing", a distance far smaller than the aperture of large astronomical telescopes ${ }^{6}$. This observation led to the interest in the astronomical community as to how sub-aperture wavefront irregularities might affect the image quality in large telescopes.

\section{APPLICATION TO ASTRONOMY}

Fried and many others applied the turbulence theories previously developed to atmospheric phenomena, both to understand how weather patterns ${ }^{4}$ evolve and to how the atmosphere limits the quality of the wavefronts passing through it. This second application leads directly to the understanding that for telescope apertures greater than the Fried parameter of the local atmosphere are not limited in performance by diffraction, but by the random perturbations of the incoming wavefront. This in turn leads to a way to specify primary mirrors in such a way that the mirror is not the limiting factor in performance, but at the same time, is not needlessly overly specified, increasing the time and cost of manufacture.

Another related application is in observatory site selection. While there are many factors involved, it is convenient to have a single number that represents the "seeing" at various sites and times. The best sites have dark skies, low humidity, and prevailing laminar flows, as opposed to highly turbulent flows of the air above the dome.

The first example of the SF as a specification was made by David Brown in 1983 for the 4.2 meter William Herschel Telescope ${ }^{7}$, produced by Grubb Parsons in England. His analysis showed that an optimal spec for this mirror was $\mathrm{r}_{0}=50$ $\mathrm{cm}$, which was specified at only four separations, 2, 8, 32, and $128 \mathrm{~cm}$. Many variations on this idea have been used since then as a part of large mirror specifications, including a continuous spec, for which the data must fall beneath the spec curve at all separations. Modifications to the structure function way of specification have been made to account for the effects of instrument noise at low separations and for improvements in telescope tracking at large separations. 


\section{NEED FOR A SPATIAL SCALE FIGURE/ROUGHNESS SPECIFICATION FOR OPTICS MANUFACTURE}

From Sections 2 and 3, it is clear why in the large optics field it is necessary to specify wavefront quality as a function of the mirror diameter. For more modest size optics this was not much of a concern in the past because optics were ground and polished using full size, rigid tools that produce nearly perfect spherical surfaces and a well behaved, or at least predictable, optical behavior.

Because of the use of spherical surfaces in the past it was safe to use a single number designation for the figure of optical surfaces, and this type designation went along well with the practice of testing figure against a test glass and observing Newton's rings as a measure of radius match and deviation from a pure spherical shape. The deviation from spherical was given as a peak-to-valley number in terms of fringes of deviation.

Once interferometers were used for figure testing it became obvious that the peak-to-valley specification was too severe because there were always outlier points (real or noise) in an interferogram that gave a large peak-to-valley when in fact the vast majority of the surface was well within spec. This led to a change in specification to a single number rms value for the figure, or an agreed upon number of points that could be ignored when deriving a peak-to-valley number ${ }^{1}$. Most interferometer data analysis programs now have a means of limiting outlier data from the peak-to-valley or rms values calculated.

Even with these changes to figure specifications driven by the better testing methods afforded by interferometers there are cases where the optics clearly met the rms specification but performed poorly in their ability to produce good images $^{3}$. In addition, optical designs were incorporating more and more aspheric surfaces that could not be produced with rigid tools but had to be made with smaller, flexible tools that often left zones and other perturbations to the desired "smooth" surface ${ }^{8}$ that had bad effects on optical performance ${ }^{2}$.

Partially as a result of optics being made to a single number specification for figure, standards were written by ISO Technical Committee 172 Optics and Optical Instruments beginning in about 1990 to help address these problems. In particular, the standard ISO 10110-8 on Texture ${ }^{9}$ addresses surface roughness and gives several methods of specifying it including a Power Spectral Density (PSD) function in a power law form as PSD $=\mathrm{A} / f^{\mathrm{B}}$ where the PSD had units of $\mathrm{nm}^{2}$ per cycle/mm, A and B are constants, and $f$ is the spatial frequency in cycles $/ \mathrm{mm}$.

Unfortunately, at the time the initial standard was being written there were legacy issues and the idea of applying the PSD to the entire surface rather than just a small piece of it was not addressed. Instead, another section of the standard, 10110-14, on wavefront deformation and a whole new standard, ISO14999, on interferometric measurement of optical elements and systems were written but did not include a PSD method of specification, only single number specifications. This omission does not in any way take away from the countless hours the members of the committees put in to write and promote better methods of specifying more complex surfaces. It is a good beginning and the work is ongoing under the direction of ISO, and in the US of Dave Aikins and the OEOSC?

\section{IMPLICATIONS OF A POWER LAW SPECIFICATION FOR FIGURE AND FINISH}

Given that there is a method outlined in the ISO 10110-8 standard on surface texture, that is, surface roughness as it is usually referred to, we want to explore some of the implications of specifying figure and finish in this way. First of all, we are going to treat the surface height errors as isotropic in this discussion to keep things simple. For the more general 2D case see the excellent, relatively recent paper by He, et. al. ${ }^{10}$ that includes many references that give a historical perspective to the discussion in this paper and lists the work of some of the authors who have contributed ideas on specifications for optics other than large scale mirrors. 
Probably the most important implication of the power law is that it is a simple way of characterizing a surface relative to the spatial scale of the surface, or distance between points where the surface height is measured. It depends on only two parameters, a constant A that is an indication of the "smoothness" of the surface and a constant B that tells how fast the surface gets smoother as a function of the distance between points of measurement of the height.

Second, it has been shown ${ }^{11}$ that over a huge range of spatial distances almost all surface height distributions obey a power law with the constant $\mathrm{B} \approx 2$. Thus a power law seems an appropriate and simple way to characterize an optical surface all the way from its full diameter to the microscopic scale commonly referred to as roughness or texture.

Third, some care must be used when applying the power law because it only strictly applies to surfaces that are statistically stationary, (or at least with stationary increments), that is, subsections of the whole surface are similar to one another. Clearly an optical surface with some figure error, say astigmatism, is not the same in all sub-regions as some are concave and some convex, relative to the best-fit sphere. As we will show by finding the structure function for a particular array of data, the structure function does represent the surface while the power law representation does not at the long separation end of the spatial scale because the surface is not stationary.

\section{SPATIAL SCALE NORMALIZATION OF THE STRUCTURE FUNCTION}

Up to now we have talked about applying the structure function description of the figure and roughness of a surface over the whole diameter of the optic. Certainly this applies over the first element, or entrance pupil, of an optical system but as the light focuses down to an image, surfaces toward the image plane have a smaller and smaller beam footprint as the light converges on the image plane. This means that the spatial scale of the full diameter used to evaluate the SF should be scaled as the footprint of the beam as it goes through the optical system ${ }^{12}$.This is analogous to the idea that a scratch on an objective lens of a binocular has little impact on the optical performance while a scratch on the object plane of the eyepiece is clearly visible and distracting to the user. This suggests that it may make sense to normalize the SF in terms of the diameter of the whole surface over which it is evaluated rather than using a spatial scale in units of length such as $\mathrm{mm}$.

\section{DETERMINATION OF THE SF AND FIT OF THE POWER LAW TO THE SF}

The structure function is defined, as in Eq. 1, as $S F(r)=\left\langle\left[z\left(r+r^{\prime}\right)-z\left(r^{\prime}\right)\right]^{2}\right\rangle$ where $r^{\prime}$ is any point in the aperture and $r$ describes another point a distance $r$ from $r^{\prime}$, the $z$ are the heights at $r^{\prime}$ and $\left(r+r^{\prime}\right)$, and the brackets indicate the ensemble average over all $r^{\prime}$. One way of finding the SF is to do a 2D Fourier transform over all the data in the aperture. This method is used by most interferometer data analysis programs but the details of the method remain a black box and the units of $\mathrm{nm}^{2}-\mathrm{mm}$ and $\mathrm{mm}^{-1}$ are somewhat hard to visualize.

We have used a random number generator to find pairs of x,y and x',y' within the aperture and then find the square of the height difference at these points ${ }^{13}$. This method, using about $10^{6}$ point pairs on a megapixel grid of data and about 20 bins takes only a few seconds to calculate. Whether using the Fourier or random number method, the array of data must be detrended to remove tip and tilt. Figure 1 shows the concept expressed above for one set of points where we use an array of data 1536 pixels square.

This same method is used to find $r$ and $\Delta z^{2}$ for a large number of point pairs within the array, typically about $10^{6}$ pairs of points, for a 1 to $4 \mathrm{Mp}$ array of data. This will give a smooth curve when separated into 20-25 bins of varying $r$ values. Here there is plenty of data so missing data points can simply be ignored and there is no constraint on the shape of the aperture in general, but since we are assuming isotropy, circular or square arrays are necessary to meet that assumption. Figure $1 \mathrm{~b}$ is a height map of the surface we use in this example. 

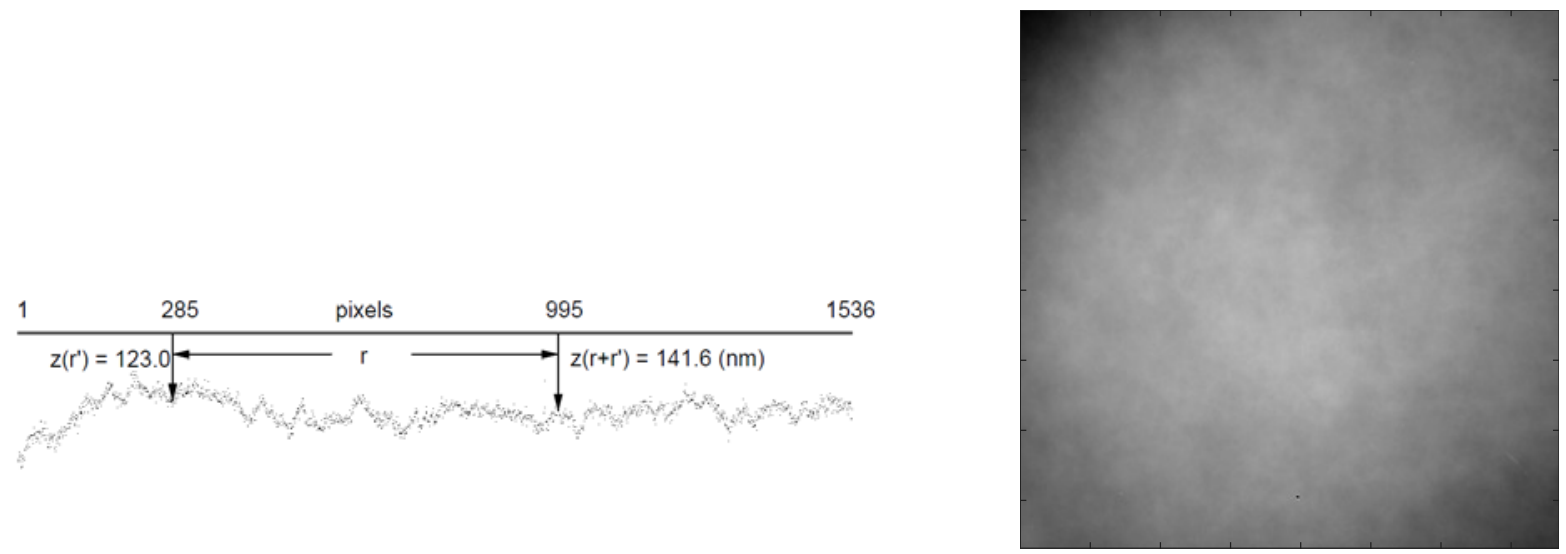

Figure 1 (a) A typical slice of the data set showing a pair of points where their heights are measured relative to a plane reference, and (b) the height map of data used in this example. The gray bar is in nm with an array size of 1536 pixels square.

Once the data pairs $\left(r, \Delta z^{2}\right)$ are extracted from the map they are sorted by $r$ and binned. Then the average value of $\Delta z^{2}$ in each bin is calculated and a plot made of $\left\langle\Delta z^{2}(r)\right\rangle$, the SF, versus $r$. Some thought must go to assigning widths to the bins. If the bins are evenly spaced far too much data is gathered in the bins at the long scale of $r$. Since the expected power law result is usually plotted on a log-log scale, assigning bin separations as the $\log _{10}$ of $r$ makes sense, but even here the short $r$ end of the scale is starved for data. The approach we have chosen is to scale the bin widths by $r^{2}$ so they are proportional to equal areas in the array. Figure 2 shows the effect of this $r^{2}$ binning versus the $\log _{10}(r)$ binning. While the longer $r$ distances again dominate at least the shorter $r$ distances are not starved for data and subject to variability due to a small sample size.

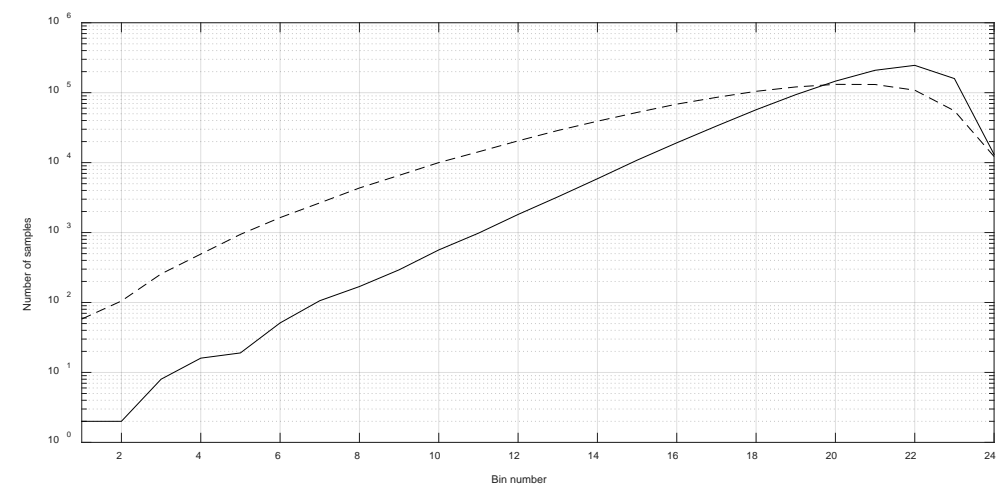

Figure 2 Number of samples in each bin with log spacing (solid) and with bin width proportional to $r^{2}$ (dashed).

When the SF is plotted using linear scales the SF looks like Figure 3. This is not the way the SF is usually plotted but some features are easier to see in this form. First, the maximum of the SF is about $r=900$ pixels. Looking at Figure $1 \mathrm{~b}$ this is about the average distance in pixels from the center of the height array to the edge (which, on average, is the maximum surface error difference in this case).

The downward hook at the longest separations is because the heights at the edge of the array are all roughly the same (at least relative to the center of the array) so when the heights at two points near the extremes of the array are differenced, the result is smaller than the height difference between the center and the edge. If the overall power in the height array had been removed before doing the analysis, the SF curve would have still reached its maxima at about 900 pixels and but the slope of the SF would have been less because the contribution due to power would have been removed. 


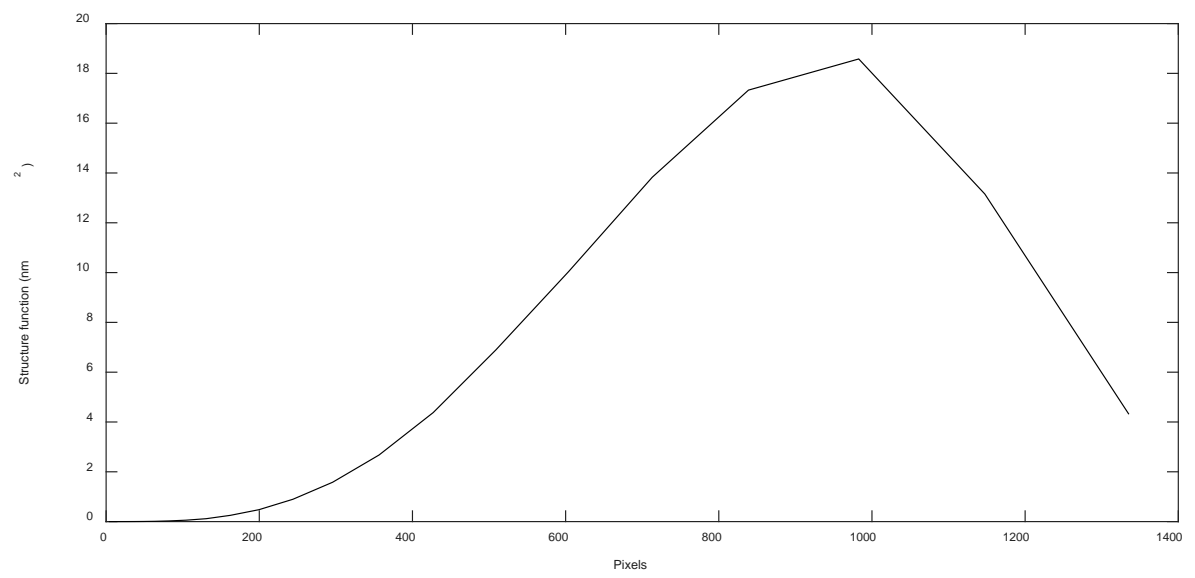

Figure 3 Example SF plotted with linear scales, with separation in pixels and the SF in $\mathrm{nm}^{2}$.

The power and other low order aberrations were not removed to use this as an example of why, for surface roughness, it gives a truer answer to the "roughness" if, what we tend the think of as figure errors, are removed first. It also is an example of why the surface is non-stationary, and the effect this has on the power law approximation. Figure 4 shows the same data SF plotted on a log-log scale and shows the roughly straight line form we expect from power law behavior. We have also changed the scale of the data to $\log _{10}(\mathrm{SF})$ and $\log _{10}(r)$ so we can easily fit a straight line of the form $\log _{10}(\mathrm{SF})=2.756 *\left(\log _{10}(\right.$ pixels $\left.)\right)-6.885 \mathrm{~nm} \wedge 2$.

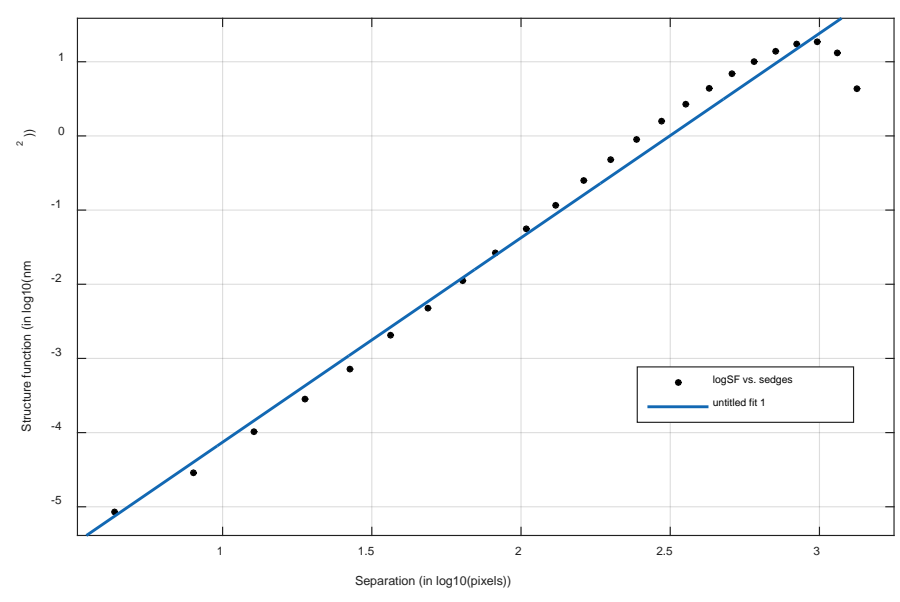

Figure 4 Example SF plotted on log-log scale.

It is now immediately clear that a large amount of information about the surface was lost in the linear representation at the small separation part of the graph. In fact, the SF varies by about 6 orders of magnitude over the 1536-pixel array. Further it is clear that the SF is nearly linear when plotted this way and this implies that a power law is a good approximation to the SF, that is, the SF is proportional to the separation of data points raised to a power. Also, the $r^{2}$ method of binning we used does not give evenly spaced bins in log space but it is a good approximation.

If we now fit a straight line to the data, we get a picture as in Figure 4 where we have changed the units to $\log _{10}$ (pixels) and $\log _{10}(\mathrm{SF})$ in order to get a linear fit of SF $=2.756 *\left(\log _{10}(\right.$ pixels $\left.)\right)-6.885 \mathrm{~nm}^{2}$. Notice the fit is quite good even 
though there was some residual power in the array of height data, and would have been better had the power and low order aberrations been removed first, that is, if the surface really had stationary increments.

To find the power law coefficients there is a little algebra. The B exponent in the power law is the slope of the linear fit, namely, 2.756. To find A we evaluate the SF at some separation by taking the antilog of the logSF and divide by the same separation raised the power $\mathrm{B}$, or

$$
A=\frac{S F(3)}{r(3)^{b}}=\frac{24.15}{1000^{\wedge} b}=\frac{24.15}{1.78 \times 10^{8}}=1.37 \times 10^{-7} .
$$

This gives $\mathrm{SF}=1.37 \times 10^{-7} * r^{2.76} \mathrm{~nm}^{2}$. Since surface roughness is usually specified in terms of rms in nm we simply take the square root of the SF (hereafter, RSF) to get rms $=3.69 \times 10^{-4} * r^{1.375}$. We can also get a curve for the rms slope variation with separation by dividing by $r$ to get rms slope $=3.69 \times 10^{-4} * r^{(1.375-1)}=3.69 \times 10^{-4} * r^{0.375}$. The curves derived from the SF and RSF and rms slope are displayed in Figure 5.

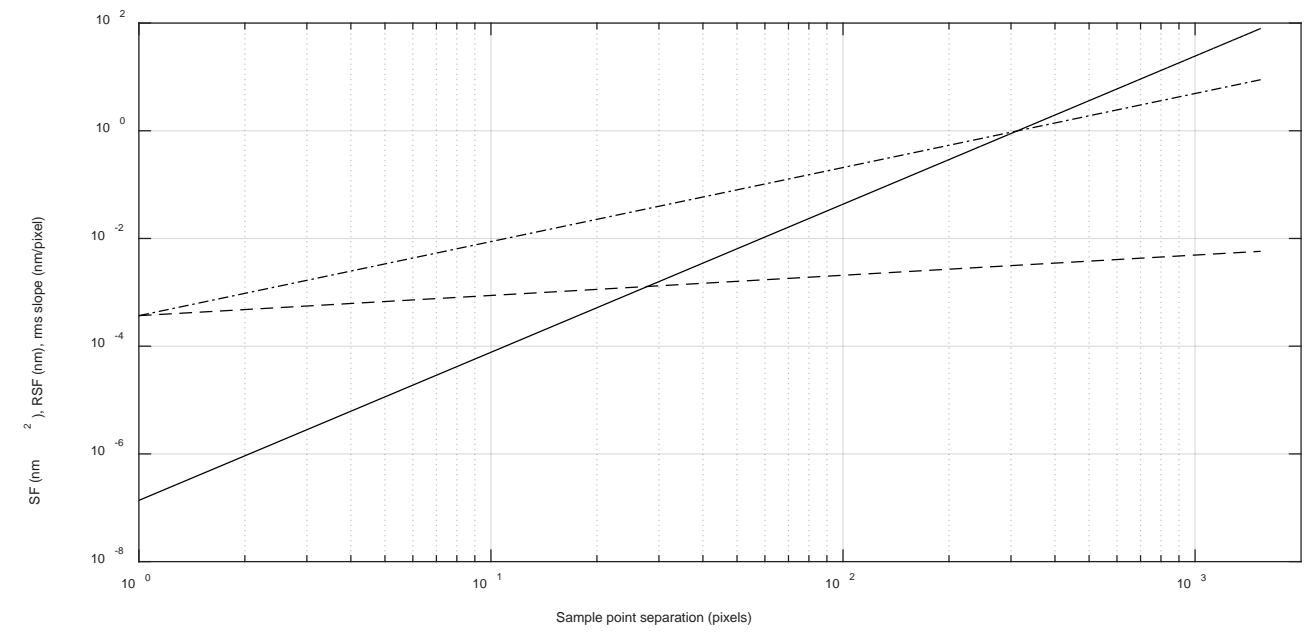

Figure 5 The Structure function (solid), its square root, the RSF, (dot-dash) and rms slope (dash) vs. separation of data points in pixels.

Because we have used pixels as the units of point separation the slope is given in terms of nm/pixel. For a surface of this smoothness the slopes in real units would be on the order of $\mu$ radians or less. What is unusual here is that the slopes decrease as the separation gets shorter, something that is counter-intuitive until you consider that the height array in Figure $1 \mathrm{~b}$ we started with had some low order aberration, mainly power. Because of this, as the separation gets smaller we can expect the rms slope to get smaller rather than larger if the power had first been removed.

In fact, since we subtract 1 from the exponent of the rms power law to get the slope, that is, we divide by the separation $r$, it becomes clear that for the rms slopes to get larger with shorter separation, the exponent of the rms error must be less than 1 . This in turn means that the exponent of the SF should be less than 2. In an ideal world where the rms slope neither increased nor decreased with a change in separation, the exponent on the SF power law would be exactly 2, something that has generally been found to be true for natural surfaces regardless of scale ${ }^{11}$. 


\section{RELATIONSHIP OF THE PSD TO THE SF}

There are two issues left to comment on; units and the hook at the long separation end of the SF. We have been rather sloppy in terms of units. In the ISO standard and most interferometer software the PSD is in units of $\mathrm{nm}^{2}$ per cycle/mm while the abscissa is spatial frequency in cycles $/ \mathrm{mm}^{-1}$. This shows that the area under the PSD curve is the single number variance, $\sigma^{2}$, in $\mathrm{nm}^{2}$. When the $\mathrm{SF}$ is plotted the $\mathrm{SF}$ is in units of $\mathrm{nm}^{2}$ while the abscissa is in units of $\mathrm{mm}$, a unit of measure easier to visualize than cycles/mm. Even more conveniently, the RSF is in units of $\mathrm{nm}$ rms vs. spatial scale.

The second issue is the departure from straight line behavior of the SF and how this should be treated when making the power law approximation. The peak in the SF comes at a separation somewhat greater than half the maximum possible separation. This is a separation that is ordinarily associated with figure, or the spatial scale of low order aberrations. As is suggested in a previous paper ${ }^{3}$ it is probably best to fit the straight line to the SF from the half aperture point at the long separation end and specify that the SF remain under this value on out to the maximum separation.

At the short separation end of the SF, the SF will rise above the straight line fit due largely to instrumental and algorithmic deficiencies such as a drop off in spatial resolution of the instrument and the finite pixel size of the detector. Again these shortcomings are probably best dealt with by pinning the short separation end of the straight line to where the SF rises from a straight line behavior and making the specification a flat line from this point on down to the 1 pixel separation limit. This gives a slope of 2.74 and a power law PSD of $3.68 \times 10^{-7}$ pixels $^{2.47} \mathrm{~nm}^{2}$, or in rms terms $6.06 \times 10^{-4}$ pixels $^{1.47} \mathrm{~nm}$, not very different than the result obtained earlier. This fitting approach is shown in Fig. 6.

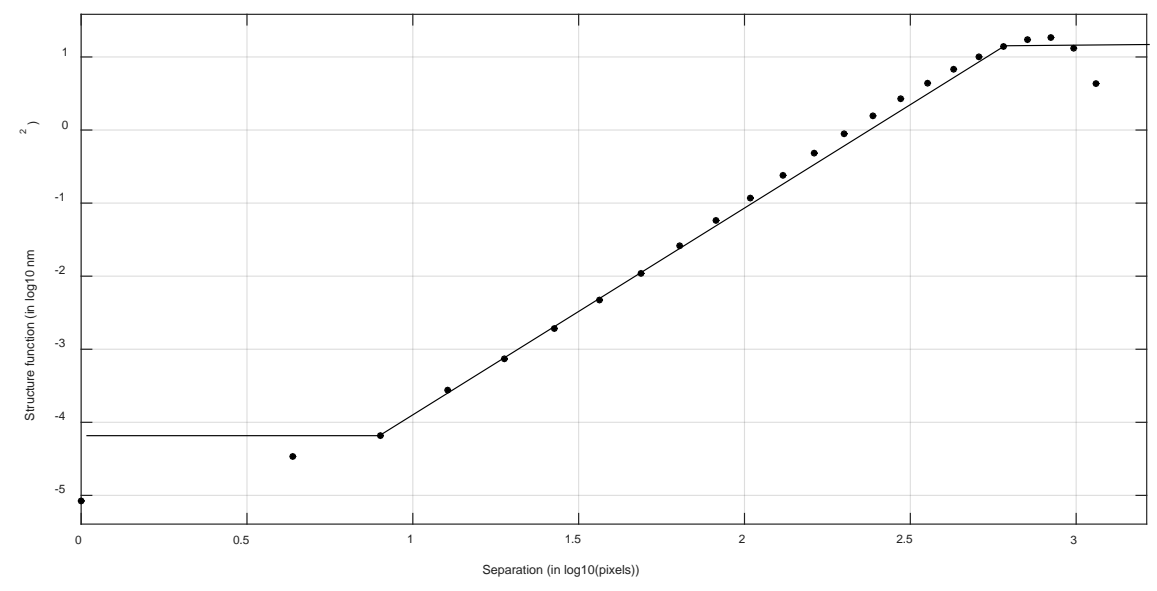

Fig. 6 SF curve from Fig. 5 with a power law curve shown in the straight line portion of the SF and a flattened portion at either spatial extreme.

\section{CONCLUSIONS}

We have shown how the structure function and power spectral density are useful metrics for analysis of residual surface errors, and that the power law form of a specification can easily be compared with measured interferometric data. While the strict formulation of the PSD is useful, the units are non-intuitive, in contrast to the RSF which has units of rms error vs spatial separation. While the SF has been applied as a spec to several large optics fabrication projects, it has not been as widely used in the small optics community thus far. We hope that some of the ideas in this paper may encourage the wider use of the power law SF and PSD in the specification of optical surfaces. 


\section{REFERENCES}

[1] Evans, C.J., "PVr - a robust amplitude parameter for optical surface specification,” Opt. Eng. 48, 043605 (2009).

[2] Forbes, G.W.,” Never-ending struggles with mid-spatial frequencies," Proc. of SPIE 9525, 95251B (2015).

[3] Parks, R.E., “Specifications: Figure and finish are not enough,” Proc. SPIE 7071, 70710B (2008).

[4] Richardson, L.F., [Weather prediction by numerical process], Cambridge University Press, (2007, reprint from 1922).

[5] Kolmogorov, A.N., "The Local Structure of Turbulence in Incompressible Viscous Fluid for Very Large Reynolds Numbers", (1941).

[6] Fried, D.L., "Statistics of a Geometric Representation of Wavefront Distortion,” JOSA, 55, 1427-1431 (1965).

[7] Brown, D.S., “Optical Specification of Ground Based Telescopes,” Proc. SPIE 399, 12-14 (1983).

[8] Tuell, M.T., Burge, J.H. and Anderson, B., “Aspheric optics: smoothing the ripples with semi-flexible tools,” Opt. Eng. 41(7), 1473-1474 (2002).

[9] ANSI at www.ansi.org and OEOSC (Optics and Electro-Optics Standards Committee) at www.oeosc.org.

[10] He, L., Evans, C.J. and Davies, A., "Two quadrant area structure function analysis for optical surface characterization,”OPT. EXP., 20, 23275-80 (2012).

[11] Sayles, R.S. and Thomas, T R., "Surface topography as a non-stationary random process,” Nature, 271, 431-34 (1978).

[12] Tamkin, J.M. and Milster, T.D., "Effects of structured mid-spatial frequency surface errors on image performance," Appl. Opt., 49, (2010).

[13] Zhelem, R., "Specification of optical surface accuracy using the structure function,” Proc. SPIE 8083, 808310 (2011).

[14] West, S., Burge, J., Cuerden, B., Davison, W., Hagen, J., Martin, H., Tuell, M., Zhao, C, and Zobrist, T., "Alignment and use of the optical test for the $8.4 \mathrm{~m}$ off-axis primary mirrors of the Giant Magellan Telescope," Proc. SPIE 7739, 77390N-15 (2010).

\section{ACKNOWLEDGEMENTS}

We would like to acknowledge roughness data provided by Wm. P. Kuhn of Opt-E used in the example in the body of the paper, and data from Richard F. Caris Mirror Lab in the last example in the Appendix. We also appreciate the insightful comments of Hubert (Buddy) Martin that have led to a better paper.

\section{APPENDIX}

\section{One example of surface roughness that affected performance}

This example shows that even the minutest details of a surface and how it is manufactured can affect performance. This sample was found to exhibit scattering in a plane when exposed to a laser beam as one would expect from a diffraction grating. Because the surface was polished by traditional polishing methods it was not believed the surface could scatter in this manner. However, when the surface was viewed with a bright field microscope and grazing illumination a structure was visible on the surface that had a resemblance to a grating running at about $45^{\circ}$ as shown in Figure A1. 

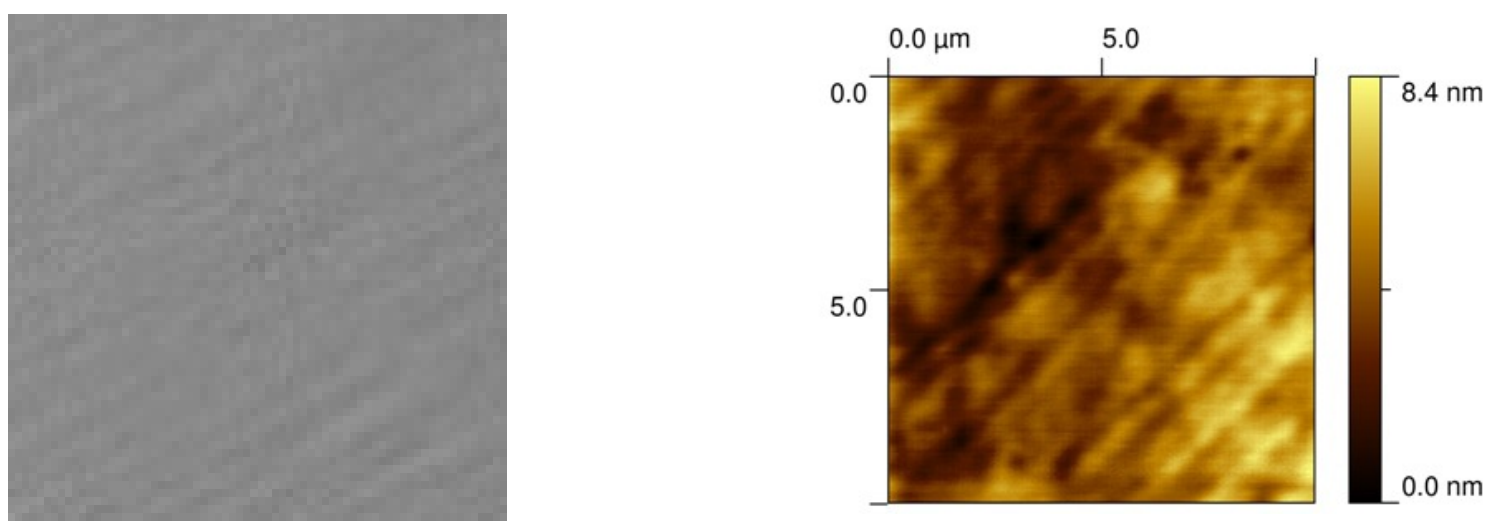

Figure A1 (a) Bright field microscope picture of a roughly $100 \mu \mathrm{m}$ square area of a sample illuminated obliquely that exhibited a grating like behavior, and (b) a surface map made with an atomic force microscope.

When this sample was available for analysis we (REP) did not have access to a surface roughness interferometer but did have access to an atomic force microscope (AFM). A $10 \mu \mathrm{m}$ square sample from the AFM is shown in Figure A1b. A power spectrum from the AFM showed a broad peak at $1.2 \mu \mathrm{m}^{-1}$ indicating a groove spacing of about $800 \mathrm{~nm}$ while the average height of the grooves was on the order of a few nm. It was hypothesized that the grooving, or grating effect, was caused at the very end of the polishing cycle when the sample was slid off the polishing lap leaving micro-grooving from polishing lap and abrasive.

\section{An example of 2D periodic structure analyzed with the SF}

During the construction of the Giant Magellan Telescope principal off-axis segment test ${ }^{14}$, a 3.75 -meter sphere, with a radius of 25.5 meters was fabricated at the Steward Observatory Mirror Lab (now the Richard F. Caris Mirror Lab) at The University of Arizona. It is a lightweight honeycomb structure cast from Ohara E6 borosilicate glass. Since this unique optic is used essentially nadir pointing, the influence of gravity on the core structure needed to be considered. To this end, a special test was performed where the mirror was tested as usual, then, contrary to normal testing procedures, the mirror was pressurized internally to the polishing pressure of 0.29 psi. The effect of this is to "puff out" the cores, leaving the ribs lower than the rest of the surface. The following map, shown in Figure A2, shows the difference between not pressurized (normal) and pressurized. The order of subtraction here is to show the ribs as high and core centers low. The scale is shown as $100 \mathrm{~nm}$ PV surface, but the typical difference between rib and core center is on the order of $30 \pm 10 \mathrm{~nm}$.

The SF was used to analyze this map in 3 ways to not only show the expected bump at roughly $100 \mathrm{~mm}$ spacing but also to show that care must be used in selecting bin sizes. In the solid curve of Figure A3, 31 logarithmically spaced bins of $100 \mathrm{~mm}$ width were used. There is a slight bump at $100 \mathrm{~mm}$ and an upturn in the curve at the longest separations due to the down edge in the map. This largest separation includes distances that are low at the very edge of the mirror but reasonably high just inside the opposite edge.

The analysis was also done with just 11 bins logarithmically spaced but still $100 \mathrm{~mm}$ wide. Since the crosses fall on top of the solid curve the lessor binning does not affect the shape of the SF curve. On the other hand, the slope of the curve is nowhere near the expected slope of 1 (because it is RSF) suggesting that something is wrong with this analysis. 


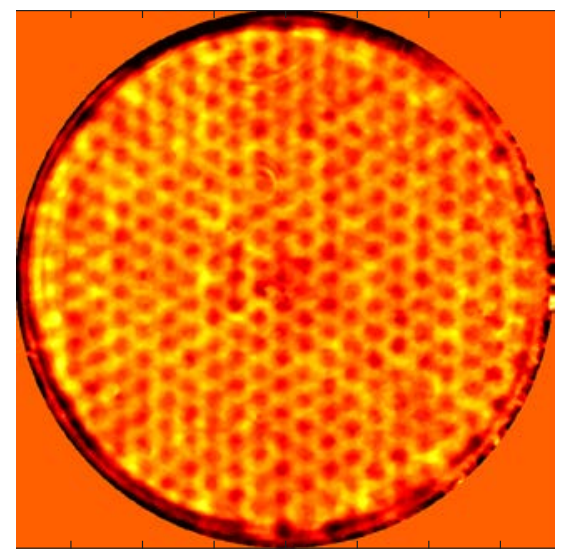

Figure A2 Difference map of honeycomb mirror under ambient testing conditions minus the same mirrror with internal air pressurization. Typical difference in height between ribs and cell centers is $30 \pm 10 \mathrm{~nm}$. Colorbar in nm surface height with low order aberrations subtracted to emphasize the core structure.

The dashed curve is the same 31 bin data where the bins were narrowed to $5 \mathrm{~mm}$ so that the fine scale features are not lost in bins whose widths are much larger than the scale of the detail in the raw data. Now when a straight line is drawn between the two ends of the curve the bump at $100 \mathrm{~mm}$ is very obvious and the expected RSF slope is much closer to 1 .

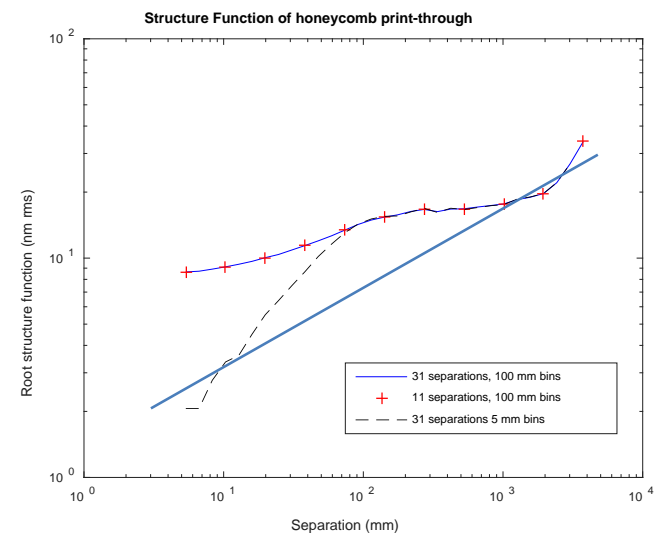

Figure A3 Root structure function of the "surface" data in Figure A2 where the solid curve is for 31 bins of 100 mm width, the crosses for 11 bins of $100 \mathrm{~mm}$ width and the dashed curve for 31 bins of $5 \mathrm{~mm}$ width. A straight line represents a power law fit to the $5 \mathrm{~mm}$ bin width data and makes the bump at $\sim 100 \mathrm{~mm}$ quite obvious. 